\title{
NOTE ON THE DETERMINATION OF SMALL QUANTITIES OF METHYL ALCOHOL.*
}

By C. SIMMONDS, B.Sc.

(Read at the Meeting, December 6, 1911.)

SxaLl proportions of methyl alcohol, especially when in mixture with ethyl alcohol, have hitherto been somewhat difficult to determine readily and accurately. Fairly good approximate results can be obtained by comparative experiments with the wellknown method of Riche and Bardy (Compt. rend., 1875, 80, 1076), or with Wolff's modification of Trillat's process (Ann. Inst. Pasteur, 1902, p. 8), but these methods are lengthy and rather troublesome. The process described by Thorpe and Holmes $(J$. Chem. Soc., 1904, 85, 1) gives good results when the quantity of methyl alcohol is

\footnotetext{
* Communicated with the sanction of the Government Chemist.
} 
not too small. It is not well adapted, however, for use when the proportion of methyl alcohol is less than about 2 per cent. of the ethyl alcohol, since the necessary subtractive correction (loc. cit., pp. 2,3 ) may in such cases equal or exceed the quantity it is desired to estimate. For determining very small proportions of methyl alcohol the method is quite inapplicable.

In such cases satisfactory determinations can be made by applying the principle of colorimetric comparison to Denigès' process for the detection of methyl alcohol (Compt. rend., 1910, 150, 832).

The possibility of thus using the process is indicated by Denigès (loc. cit., p. 833). The object of the present note is to give the procedure which the writer finds most suitable for utilising the reaction quantitatively in general analytical work-as, for example, in examining spirituous beverages, medicinal tinctures, flavouring essences, and so forth.

The alcoholic mixture is first purified, where necessary, either by the method of Thorpe and Holmes ( $J$. Chem. Soc., 1903, 83, 314), or by other suitable means. It is then diluted with water or mixed with ethyl alcohol, as the case may require, until it contains 10 per cent. of total alcohol by volume.

To 5 c.c. of this prepared liquid contained in a wide test-tube are added 2.5 c.c. of permanganate solution ( 2.0 grms. $\mathrm{KMnO}_{4}$ per 100 c.c.), and then 0.2 c.c. of strong sulphuric acid. When the reaction has proceeded about three minutes, 0.5 c.c. of oxalic acid solution is added ( 9.6 grms. crystallised acid per 100 c.c.). On shaking, the liquid becomes clear and nearly colourless. One c.c. of strong sulphuric acid is now run in and well mixed with the solution, which is finally treated with 5 c.c. of Schiff's reagent. A violet colour is developed in the course of a few minutes unless mere traces of methyl alcohol were present, when twenty or thirty minutes may be required.

This colour is due, of course, to the reaction of the fuchsine solution with formaldehyde, produced by the oxidation of the methyl alcohol. A sufficient quantity of sulphuric acid is present to prevent the development of colour with any acetaldehyde formed from the ethyl alcohol during the oxidation.

A preliminary experiment carried out as described serves to detect the presence of methyl alcohol (if this is not already known) and to give some idea of the quantity. According to the indications thus obtained, another part of the prepared liquid is further diluted, if necessary, with ethyl alcohol of 10 per cent. strength until it contains from 0.001 to $0.004 \mathrm{grm}$. of methyl alcohol in 5 c.c. ; and the experiment is repeated side by side with two or more standards for comparison. These contain $0.001,0.002,0.003$, etc., grm. of methyl alcohol in 5 c.c. of 10 per cent. ethyl alcohol. The colours produced are compared in small Nessler-tubes (25 c.c.) or in a suitable colorimeter.

With properly sensitive Schiff's reagent, $0.0003 \mathrm{grm}$. methyl alcohol in the 5 c.c. of liquid taken is readily detected. The best depths of colour for comparison, however, are given by the formaldehyde produced in the manner described from quantities of 0.001 to 0.004 grm. of methyl alcohol.

It is convenient to keep a standard solution ( $1 \mathrm{grm}$. per litre) of methyl alcohol in 10 per cent. ethyl alcohol. This is diluted as required with 10 per cent. alcohol 
to form the standards for comparison. This proportion of ethyl alcohol (10 per cent.) is a suitable strength for general work, as the distillates ordinarily obtained are stronger, and can thus be diluted down instead of having to be concentrated.

The process has the advantages of (1) being rapidly executed, (2) requiring only a small quantity of material, and (3) being directly applicable to weak distillates. The degree of accuracy obtainable is shown by the following results of a typical series of experiments :

Grm. Methyl Alcohol per 100 c.c.

$\begin{array}{cc}\text { Present. } & \text { Found. } \\ 0.005 & 0.004 \\ 0.028 & 0.029 \\ 0.044 & 0.046 \\ 0.072 & 0.072 \\ 0.100 & 0.104 \\ 0.500 & 0.492 \\ 1.000 & 0.968\end{array}$

Formaldehyde, of course, must be absent from the unoxidised solution of alcohols, or else its effect must be determined and allowed for. Glycerol must also be absent.

Government Laboratory, LONDON.

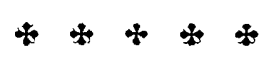

